\title{
Remarks on Mathematical Structure of BRST Theories
}

\author{
S. S. Horuzhy and A. V. Voronin \\ Steklov Mathematical Institute, SU-117966 Moscow, GSP-1, USSR
}

\begin{abstract}
A number of rigorous results concerning the mathematical structure of quantum systems with the BRST symmetry are presented. For studying model-independent properties of such systems, a generalization of the Strocchi-Wightman theory is suggested, which is a Wightman type theory in the Krein space with an $\mathrm{Op} J^{*}$-algebra of physical and ghost fields. It is proved that the BRST charge operator $Q$ is either bounded with $\sigma(Q)=\{0\}$ or unbounded with $\sigma(Q)=\mathbb{C}$. A number of conditions equivalent to the "no-ghost theorem" (which states that the kernel of $Q$ is non-negative) are derived.
\end{abstract}

BRST-symmetry which was first discovered in QCD as a certain specific symmetry of its Lagrangian, is now considered as a key structural property in a very large class of physical systems. However, we are still very far from a complete understanding of the structure of the BRST formalism as such so that all the remaining problems would be reduced to its application to new theories and models. As is always the case with describing gauge fields or dynamical systems with constraints, the construction of the formalism is not unambiguous, and different versions of the latter are possible (and indeed have been presented more than once) for the same system. To start with, already on a quite general level there are two kinds of the BRST formalism: the Kugo-Ojima method based on the Lagrangian framework and the method of quantization according to BatalinFradkin-Vilkovisky (BFV) based on the Hamiltonian framework. Although they can both be interpreted from an unified viewpoint within the continual integral framework (with ghosts introduced by means of the Faddeev-Popov procedure) and so should not have significant differences of principle, in practice they do not produce the same formulas and may not lead to the same conclusions. But comparison of different variants of the BRST formalism as well as a general assessment of it are today hindered by the shortage of rigorous results concerning its mathematical structure, both general and in models. This structure is still rather little studied, and arguably the least studied aspect of it is that concerning specific properties of operators in indefinite metric spaces. This includes, in the first place, 
all problems related to the properties of the BRST charge $Q$ and structures in the state space associated with $Q . Q$ is an operator of a special class with no analogue in the Hilbert space: a 2-nilpotent $J$-self-adjoint (or $J$-symmetric) operator in some $J$-space (Krein space) $\mathscr{H}$. In any BRST theory central problems are connected with studying the kernel of the BRST charge and proving the so-called "no-ghost theorem" which states that this kernel is a non-negative lineal (i.e. linear set; the term "subspace" we keep for closed lineals) in $\mathscr{H}$. Up to now, properties of $Q$ and the structure of $\operatorname{ker} Q$ have usually been studied anew in each concrete model, and almost never (the main exception is [1]) analysed from the general mathematical viewpoint, as statements on 2-nilpotent operators in $J$-spaces.

In this note we rigorously prove a number of facts related to the operator $Q$ and the no-ghost theorem. We find that two alternative kinds of $Q$, bounded and unbounded, are possible, and give a complete description of geometrical structures in $\mathscr{H}$ associated with $Q$. Proceeding from that, we derive several conditions equivalent to the no-ghost theorem.

At first we shall outline a simple general framework which is convenient for studying model-independent properties of the BRST formalism. In general terms, this formalism is a way of replacing a degenerate dynamical system with an equivalent non-degenerate one by means of introducing fictional "ghost" degrees of freedom. As we know (see, e.g. [2]), in quantum systems with gauge fields the Faddeev-Popov ghosts $c, \bar{c}$ can be introduced as operators which (multiplied by a Grassmann number $\lambda$ ) replace $c$-number infinitesimal parameters of local gauge transformations. The ghosts are "scalar fermions," i.e. they satisfy local anticommutation relations but transform as scalars under the Poincare group, thus violating the spin-statistics theorem. Apart from this property, the ghosts are similar to physical fields, i.e. they are operator-valued distributions whose structure is model-dependent and can vary widely (in particular, the Grassmann property $c^{2}=\bar{c}^{2}=0$ may or may not be present). In this line, the BRST formalism presents itself as a certain "extended" description of systems with local gauge invariance introduced in order to turn this local invariance into a global one (or, more exactly, in order to get an equivalent global reformulation of the local gauge invariance property). Then the BRST symmetry is just the global symmetry of the extended description equivalent to the local gauge symmetry of the initial ghostless description. If seen like this, the BRST formalism can naturally be extended from Lagrangian theories to general field theories of the Wightman type or, more precisely, of the Strocchi-Wightman type, since it deals with gauge theories in indefinite metric spaces. It is quite natural to consider a general StrocchiWightman theory complemented with scalar fermion ghost fields $c(f), \bar{c}(f)$ and enjoying the BRST symmetry which is now defined as a certain global transformation of the complete set of physical and ghost fields with the 2-nilpotent generator $Q$. Such extended Strocchi-Wightman theories share the main advantage of BRST theories: the non-negative physical subspace $\mathscr{H}^{\prime}$ which one always looks for in indefinite metric theories, now takes an extremely simple and compact form, $\mathscr{H}^{\prime}=\operatorname{ker} Q$. (It is more natural to call $\mathscr{H}^{\prime}$ the "prephysical" subspace since it is not $\mathscr{H}^{\prime}$ but some positive quotient space that is considered as the subspace of true physical states.) It is also a good thing that string theories can be included into such framework, too. 
Thus let us consider a "general BRST theory" defined as a Strocchi-Wightman theory complemented with the Faddeev-Popov ghosts. Such theory is given by a system of physical fields $\Pi(f)$ and ghost fields $c(f), \bar{c}(f)$ generating the polynomial algebra $\mathscr{P}$ of unbounded operators with a common invariant dense domain $\mathscr{D}$ in an indefinite metric space $\mathscr{H}$. [In particular, $\Pi(f)$ may be Yang-Mills fields $A_{\mu}^{a}(f)$, fields of matter $\varphi_{i}(f)$ or coordinates $X_{\mu}(f)$ of a first quantized string.] Concretization of the test function class will not be needed. The space $\mathscr{H}$ with no loss of generality [3] can be considered as a $J$-space (Krein space), i.e. it possesses indefinite $\langle$,$\rangle and Hilbert ( , ) inner products connected via the metric operator J$ such that

$$
\langle\cdot, \cdot\rangle=(\cdot, J \cdot) ; \quad J=P_{+}-P_{-} ; \quad P_{+}+P_{-}=I, \quad \operatorname{dim} P_{ \pm} \mathscr{H}=\infty
$$

(all inner products are required to be linear in the second argument; if $\operatorname{dim} P_{-} \mathscr{H}<\infty, \mathscr{H}$ is the Pontryagin space, by definition). The pair $(\mathscr{P}, \mathscr{D})$ is an $\mathrm{Op} J^{*}$-algebra in the terminology of [3], i.e. an Op*-algebra with respect to the involution defined by the indefinite form in $\mathscr{H}$ as $A \rightarrow A^{+}=\left.A^{0}\right|_{\mathscr{D}}, A^{0}$ being the $J$-adjoint operator of $A \in \mathscr{P}$. Ghost operators are $J$-symmetric, $c(f) \subset c(f)^{0}$, $\bar{c}(f) \subset \bar{c}(f)^{0}$.

In the approach we follow, the BRST formalism is a description of a certain symmetry. The most general way of formulating a symmetry property is to define it as a derivation on the field algebra. Thus our starting point is the definition of the BRST symmetry as a derivation $\delta$ on $(\mathscr{P}, \mathscr{D})$. Let $A, B$ be two monomials in ghost and physical field operators. Then $\delta$ is a linear mapping of $\mathscr{P}$ into $\mathscr{P}$ such that

$$
\delta\left(A^{+}\right)=\varepsilon_{A} \delta(A)^{+} ; \quad \delta(A B)=\delta(A) B+\varepsilon_{A} A \delta(B) ; \quad \delta^{2}=0 .
$$

Here $\varepsilon_{A}=(-1)^{n(A)}, n(A)=n_{c}(A)-n_{\bar{c}}(A)$ is the difference between the numbers of the ghost $c$ and antighost $\bar{c}$ operators in the monomial $A$. So $\delta$ is a nilpotent superderivation with the domain $\operatorname{dom} \delta=\mathscr{P}$. The last property does not mean that $\delta$ is a bounded derivation since $\mathscr{P}$ is not the Banach space. A superdeviation $\delta$ on the Op $J^{*}$-algebra $(\mathscr{P}, \mathscr{D})$ satisfying conditions (1) is called the BRST transformation (cf. [4]). We assume that the BRST transformation possesses a generator, i.e. there is in $\mathscr{H}$ an operator $Q$ such that for any monomial $A$ in $\mathscr{P}$ (or possibly in some subalgebra $\widehat{\mathscr{P}} \subset \mathscr{P}$ dense in $\mathscr{P}$ in some topology) on the domain $\mathscr{D}$ (or possibly on some dense lineal $\hat{\mathscr{D}}(\mathscr{D})$ one has

$$
\delta(A)=i[Q, A\},
$$

where $[Q, A\} \equiv Q A-\varepsilon_{A} A Q$ is the supercommutator. In a typical case, when $\mathscr{P}$ has a cyclic vector $\Omega$, the equivalent definition of the generator is

$$
Q A \Omega=-i \delta(A) \Omega \text { for all } A \in \widehat{\mathscr{P}} .
$$

Indeed, it is obvious due to $\delta(I)=0$ that (3) is equivalent to (2) on the domain $\hat{\mathscr{D}}=\hat{\mathscr{P}} \Omega$ and with the additional condition $Q \Omega=0$.

In the general case, the existence of the generator is a restriction on the theory because (3) is the correct definition of an operator only if

$$
A \Omega=0 \text { implies } \delta(A) \Omega=0 \text { for any } A \in \hat{\mathscr{P}} \text {. }
$$


However, (4) can often be proved. It is clear, for instance, that (4) is fulfilled if $\Omega$ is separating for $\mathscr{P}$. This property holds in a majority of systems with local structure, i.e. with a net of local subalgebras $\mathscr{P}(\mathcal{O}) \subset \mathscr{P}$, for $\widehat{\mathscr{P}}=\mathscr{P}_{\text {loc }} \equiv \bigcup \mathscr{P}(\mathcal{O})$ (local regions $\mathcal{O}$ in some theories may be not from the Minkowski space). In field theory this property follows from the Reeh-Schlieder theorem which is valid in the StrocchiWightman theory if the states of the form $\Pi\left(x_{1}\right) \ldots \Pi\left(x_{n}\right) \Omega$ satisfy the spectrum condition (i.e. their Fourier transforms in the translation-invariant variables $\xi_{i}=x_{i}-x_{i+1}$ have supports in the closed forward light cone $\bar{V}_{+}$). But validity of the spectrum condition is not evident at all in BRST theories. Even in the usual Strocchi-Wightman theory, when the spectrum condition is imposed on neutral and negative state vectors, this is justified only by rather loose arguments from the perturbation theory and scattering theory [5]. And if the states include ghosts, the spectrum condition for them is physically groundless (at least, in the ghost variables), although it is sometimes assumed for practical needs (e.g. in [6]). What is more, the presence of tachyons in the string theory shows that the spectrum condition can really be violated in BRST schemes. One can now see that the ReehSchlieder theorem, and hence the existence of $Q$, can be proved as well if the spectrum condition is only required of states from some dense lineal $\widehat{\mathscr{D}} \subset \mathscr{D}$. Hence it is sufficient to assume that ghostless states (satisfying the spectrum condition) are dense in $\mathscr{H}$. But this assumption is also unrealistic since ghosts have their own degrees of freedom and very often the state space is of the form $\mathscr{H}=\tilde{\mathscr{H}} \otimes \mathscr{H}_{g}, \mathscr{H}_{g}$ being the space of ghost states. Thus in an arbitrary BRST theory the existence of the generator for the BRST transformation (1) is an additional assumption, and if $\widehat{\mathscr{P}} \neq \mathscr{P}$, then the same is true about the existence of a dense domain for the operator $Q^{2}$. Under these assumptions, $\delta$ has the generator $Q$ (the BRST charge) which is a 2-nilpotent operator. It is easy to give its complete mathematical characterization.

Proposition 1. 1. Let $Q$ be an arbitrary 2-nilpotent operator in the J-space $\mathscr{H}$. Then the following alternative takes place:

either $Q$ is a bounded operator and $\sigma(Q)=\{0\}$,

or $Q$ is an unbounded operator and $\sigma(Q)=\mathbb{C}$.

2. If $Q$ is the generator of the superderivation $(1)$ on $(\mathscr{P}, \mathscr{D})$ with $\mathscr{D}=\mathscr{P} \Omega$, then $Q$ is $J$-symmetric if and only if

$$
\langle\Omega, \delta(A) \Omega\rangle=0 \quad \text { for any } \quad A \in \hat{\mathscr{P}} .
$$

3. Let the BRST transformation satisfy the condition (5) and its generator $Q$ be bounded. Then $Q$ is a J-hermitian definizable operator having the only regular critical point $\lambda=0$ and the trivial $J$-spectral function

$$
E_{\Delta}(Q)=0 \quad \text { for any } \Delta \subset \mathbb{R}, \quad \bar{\Delta} \not 0 ; \quad E_{\Delta}(Q)=I \text { for any } \Delta \ni 0 .
$$

Proof. 1. Let us assume that $\sigma(Q) \neq \mathbb{C}$, i.e. the resolvent set of $Q$ contains at least one point $\lambda$. Then $Q$ is closed, since $Q-\lambda I$ has the bounded inverse $R_{\lambda}(Q)=(Q-\lambda I)^{-1}$ and so is closed. Because of $R_{\lambda}(Q) \mathscr{H} \subset \operatorname{dom} Q, Q^{2}$ can be represented as

$$
Q^{2}=(Q-\lambda I)\left(Q+\lambda Q(Q-\lambda I)^{-1}\right) \text {. }
$$


Here the right-hand side is an operator of the form $T S$, where $T$ has the bounded inverse and $S$ is closed [because $Q$ is closed and $Q(Q-\lambda I)^{-1}$ is bounded]. It is easy to check directly that such an operator is closed. Closedness of $Q^{2}$ together with $Q^{2}=0$ imply that the domain of $Q^{2}$ is closed and since this domain is dense in $\mathscr{H}$, we obtain $\operatorname{dom} Q^{2}=\mathscr{H}$. Hence it follows that $\operatorname{dom} Q=\mathscr{H}$ and, by the closed graph theorem, $Q$ is bounded. Then due to [7, Chap. VII] nilpotence of $Q$ implies $\sigma(Q)=\{0\}$.

2. If $Q$ is the generator of the superderivation (1) on $(\mathscr{P}, \mathscr{D})$ with $\mathscr{D}=\mathscr{P} \Omega$, then $Q$

$$
\begin{aligned}
\langle Q A \Omega, B \Omega\rangle & =\langle-i \delta(A) \Omega, B \Omega\rangle=\left\langle-i B^{+} \delta(A) \Omega, \Omega\right\rangle \\
& =\left\langle-i \varepsilon_{B^{+}}\left(\delta\left(B^{+} A\right)-\delta\left(B^{+}\right) A\right) \Omega, \Omega\right\rangle=\varepsilon_{B^{+}}\left\langle i \delta\left(B^{+}\right) A \Omega, \Omega\right\rangle \\
& =\left\langle A \Omega,-i \varepsilon_{B^{+}} \delta\left(B^{+}\right)^{+} \Omega\right\rangle=\langle A \Omega, Q B \Omega\rangle,
\end{aligned}
$$

so that

$$
\langle Q \Phi, \Psi\rangle=\langle\Phi, Q \Psi\rangle \text { for all } \Phi, \Psi \in \hat{\mathscr{D}}
$$

i.e. $Q \subset Q^{0}$. Necessity is trivial: if $Q \subset Q^{0}$, then due to $Q \Omega=0$ one has for any monomial $A \in \mathscr{P}$ :

$$
\langle\Omega, \delta(A) \Omega\rangle=\langle\Omega, i[Q, A\} \Omega\rangle=\left\langle Q^{0} \Omega, i A \Omega\right\rangle=0 .
$$

3. At first, let us recall the basic definitions. Definizable operators studied in detail in [8] (and called positizable in [9]) are the main class of operators in $J$-spaces for which a satisfactory spectral theory was developed so far. A $J$-selfadjoint operator $A$ with the non-empty resolvent set is called definizable if there is a polynomial $p(\lambda)$ (the definizing polynomial) such that $\langle p(\lambda) \Phi, \Phi\rangle \geqq 0$ for all $\Phi \in \operatorname{dom} A^{k}, k$ being the power of $p$. If $A$ is definizable, it has a $J$-spectral function $E_{\Delta}(A)$. The latter is defined as a homomorphism $E(A): R(S) \rightarrow \mathscr{P}(\mathscr{H})$, where $S=\left\{\alpha_{1}, \ldots, \alpha_{n}\right\}$ is a finite set of points $\alpha_{i} \in \mathbb{R}, R(S)$ is the $\sigma$-algebra generated by all intervals $\Delta \subset \mathbb{R}$ for which $\alpha_{i}$ are not boundary points and $\mathscr{P}(\mathscr{H})$ is the set of all $J$-projection operators $E$ in $\mathscr{H}$ (i.e. $E^{2}=E^{0}=E$ ). The homomorphism $E(A)$ is required to be such that for any $\lambda, \mu \notin S, \mu<\lambda$ and $\mu_{\alpha} \rightarrow \mu+0$ the operators $E_{\left(\mu_{\alpha}, \lambda\right)}(A)$ converge strongly to $E_{(\mu, \lambda)}(A) ; S$ is called the set of critical points of $A$. A critical point $\alpha \in S$ is called regular if strong limits ${ }_{\lambda-\lim _{\lambda \rightarrow 0}} E_{(-\infty, \lambda)}(A)$ and $\operatorname{sim}_{\lambda \rightarrow \alpha+0} E_{(\lambda, \infty)}(A)$ exist; otherwise it is called singular. For any $\Delta \in R(S)$ one has $A E_{\Delta}(A)=E_{\Delta}(A) A ; \sigma\left(\left.A\right|_{E_{\Delta}(A) \mathscr{H}}\right) \subset \bar{\Delta}$ and if $\Delta \cap S=\emptyset$, then $A E_{\Delta}(A)=\int_{\Delta} \lambda d E_{\lambda}$.

Now, the assumptions made clearly imply that $Q$ is $J$-symmetric and definizable with the definizing polynomial $Q^{2}$. We check directly that the function $E_{\Delta}(Q)$ defined by (6) and hence having the set of critical points $s(E)=\{0\}$ satisfies the definition of the $J$-spectral function. Since we can see directly that the strong limits $s-\lim _{\lambda \rightarrow-0} E_{(-\infty, \lambda)}=0$ and $s_{\lambda \rightarrow+0} E_{(\lambda, \infty)}=I$ exist, then $\lambda=0$ is a regular critical point. Finally, since ker $Q$ includes neutral vectors (for instance, all vectors from $\operatorname{ran} Q)$, this critical point cannot be removed from the set $s(E)$. Proposition 1 is proved.

In physics $Q$ was usually treated as a bounded operator. But it is easy to point out large classes of unbounded operators $Q$ such that $Q=Q^{0}, Q^{2}=0$. Let $\mathscr{H}_{1}, \mathscr{H}_{2}$ be 
the Hilbert spaces and $\mathscr{H}=\mathscr{H}_{1} \oplus \mathscr{H}_{2}$ the Krein space with $J=\left(\begin{array}{rr}I & 0 \\ 0 & -I\end{array}\right)$. Then any operator of the form $Q=\left(\begin{array}{ll}0 & A \\ 0 & 0\end{array}\right)$ with $A$ unbounded and skew-hermitian ( $A^{*}=-A$, the involution $A \rightarrow A^{*}$ being with respect to the Hilbert metric) has the required properties. For $J=\left(\begin{array}{ll}0 & I \\ I & 0\end{array}\right)$ these properties are fulfilled if $A^{*}=A$. Another example: let us take $\mathscr{H}=\mathscr{H}_{1} \otimes \mathscr{H}_{2}$, where $\mathscr{H}_{1}$ is the infinite-dimensional Hilbert space, $\mathscr{H}_{2}$ is any Pontryagin or Krein space, and $Q=A \otimes B$, where $A$ is unbounded and self-adjoint, $B=B^{0}, B^{2}=0$. Then again $Q=Q^{0}, Q^{2}=0$ and $Q$ is unbounded. BRST operators in simple models are typically sums of such operators.

We proceed now to studying objects in $\mathscr{H}$ connected with $Q$ and with the noghost theorem. Let us denote by $\mathfrak{M}_{0}, \mathfrak{M}_{ \pm}, \mathfrak{M}_{ \pm \pm}$the sets of all neutral, nonnegative (nonpositive), positive (negative) vectors in $\mathscr{H}$ and consider the lineals

$$
\mathscr{H}_{1}=\operatorname{ker} Q ; \quad \mathscr{H}_{d}=\operatorname{ran} Q ; \quad \mathscr{H}_{p}=J \mathscr{H}_{d}
$$

(we adopt physical notations [2] referring to the interpretation of vectors from $\mathscr{H}_{p}$ and $\mathscr{H}_{d}$ as "parent" and "daughter" states). We assume at first that $Q$ is $J$-selfadjoint (usually one can secure this property in physical situations). Then $\mathscr{H}_{p}=\operatorname{ran} Q^{*}, \mathscr{H}_{1}$ is a closed set, $J \mathscr{H}_{1}=\operatorname{ker} Q^{*}$, and due to $Q^{2}=0$ we can assume without loss of generality that $\mathscr{H}_{d} \subset \operatorname{dom} Q$ (otherwise $Q$ can be extended to the whole $\mathscr{H}_{d}$ by the zero operator). Taking this into account, we have from $Q=Q^{0}$ and $Q^{2}=0$ :

$$
\mathscr{H}_{d} \subset \mathscr{H}_{1} \cap \mathfrak{M}_{0}, \quad \mathscr{H}_{p} \subset \mathfrak{M}_{0}, \quad \mathscr{H}_{p} \perp \mathscr{H}_{d}
$$

(the symbol in the last formula means the Hilbert orthogonality). Next, by [9, p. 122] $\mathscr{H}_{1}=\mathscr{H}_{d}^{[\perp]}$ (where the symbol means the $J$-orthogonal complement) whence $\mathscr{H}_{1}^{[\perp]}=\mathscr{H}_{d}^{[\perp \perp]}$ and so we have from (7) and $\mathscr{H}_{d} \subset \mathscr{H}_{d}^{[\perp \perp]}$ :

$$
\mathscr{H}_{d} \subset \mathscr{H}_{1} \cap \mathscr{H}_{1}^{[\perp]} \text {. }
$$

Introducing the "singlet subspace" $\mathscr{H}_{s}[2]$ as

$$
\mathscr{H}_{s}=\left(\mathscr{H}_{p} \oplus \mathscr{H}_{d}\right)^{\perp}
$$

(the sum of subspaces being hilbertian orthogonal) we have

$$
\mathscr{H}_{s}=\overline{\mathscr{H}}_{s}=\mathscr{H}_{1} \cap J \mathscr{H}_{1},
$$

and taking into account $\mathscr{H}_{s}^{\perp}=\mathscr{H}_{s}^{[\perp]}$ and $\mathscr{H}_{p}, \mathscr{H}_{d} \subset \mathscr{H}_{p} \oplus \mathscr{H}_{d}$ we obtain the decompositions

$$
\mathscr{H}=\overline{\mathscr{H}}_{p} \oplus \overline{\mathscr{H}}_{d} \oplus \mathscr{H}_{s}=\overline{\mathscr{H}}_{p} \oplus \overline{\mathscr{H}}_{d}[+] \mathscr{H}_{s},
$$

where the last symbol means the $J$-orthogonal sum. Hence it is clear that $\mathscr{H}_{s}$ and $\overline{\mathscr{H}}_{p} \oplus \overline{\mathscr{H}}_{d}$ are orthocomplemented. Then the equalities $\mathscr{H}_{1}=\mathscr{H}_{d}^{[\perp]}=\left(J \mathscr{H}_{p}\right)^{[\perp]}$ $=J \mathscr{H}_{p}^{[\perp]}=\mathscr{H}_{p}^{\perp}$ together with (10) imply

$$
\mathscr{H}=\overline{\mathscr{H}}_{p} \oplus \mathscr{H}_{1} ; \quad \mathscr{H}_{1}=\overline{\mathscr{H}}_{d} \oplus \mathscr{H}_{s}=\overline{\mathscr{H}}_{d}[+] \mathscr{H}_{s} .
$$

From this set of formulas one obtains very simply a series of rather illuminating reformulations of the no-ghost theorem. 
Proposition 2. For any J-self-adjoint 2-nilpotent operator $Q$, the following conditions are equivalent:

1. $\mathscr{H}_{1} \subset \mathfrak{M}_{+}, \mathscr{H}_{d}=\overline{\mathscr{H}}_{d}$;

2. $\mathscr{H}_{d}$ is a maximal neutral lineal in $\mathscr{H}$;

3. $\mathscr{H}_{d}=\mathscr{H}_{1} \cap \mathscr{H}_{1}^{[\perp]}=\mathscr{H}_{1} \cap \mathfrak{M}_{0}$;

4. $\mathscr{H}_{s} \subset \mathfrak{M}_{++} ; \mathscr{H}_{d}=\overline{\mathscr{H}}_{d}$.

Proof. $1 \leftrightarrow 2$ coincides with the Theorem 7.2 in $\left[9\right.$, p. 14], because of $\mathscr{H}_{1}=\mathscr{H}_{d}^{[\perp]}$ and $\mathscr{H}_{d}=\mathscr{H}_{d}^{[\perp \perp]} 1 \rightarrow 3$. The first equality in 3 follows from $\mathscr{H}_{d}=\overline{\mathscr{H}}_{d}$ and $(8)$, the second follows from $\mathscr{H}_{1} \subset \mathfrak{M}_{+}$by $[9$, p. 9]. $3 \rightarrow 4$. The first equality in 3 implies that $\mathscr{H}_{d}$ is closed. Next, it follows from $\mathscr{H}_{d} \oplus \mathscr{H}_{s} \subset \mathscr{H}_{1}$ and $\mathscr{H}_{d}=\mathscr{H}_{1} \cap \mathfrak{M}_{0}$ that $\mathscr{H}_{s}$ does not include neutral vectors and hence it is definite, by $\left[9\right.$, p. 4].4 $\rightarrow 1:$ if $\mathscr{H}_{s} \subset \mathfrak{M}_{++}$then (11) and $\overline{\mathscr{H}}_{d} \subset \mathfrak{M}_{0}$ imply $\mathscr{H}_{1} \subset \mathfrak{M}_{+}$.

This simple geometrical proposition gives a clear picture of various ghostremoving mechanisms which have repeatedly been discovered in BRST or Strocchi-Wightman theories in the physical language (and often using irrelevant physical assumptions). In particular, we find here the well-known "quartet mechanism" by Kugo-Ojima [2, Sect. 3.2] (it is contained in $3 \rightarrow 1$ : $\mathscr{H}_{1}$ is nonnegative if all its neutral vectors belong to "BRST-doublets", i.e. pairs of states $\Phi, \Psi$ such that $Q \Phi=\Psi$; the necessity of the first equality in 3 went unnoticed); "the lemma on the absence of singlet ghosts" [2, Sect. 3.1] (see Corollary 3 below); etc.

As a rule, the case $Q=Q^{0}$ is sufficient for physical needs. Therefore, we shall only briefly mention the changes arising when $Q \subset Q^{0}$. The definitions of $\mathscr{H}_{1}, \mathscr{H}_{p}, \mathscr{H}_{d}$ are the same, $\mathscr{H}_{1}$ is still closed (if $Q$ is closed) and the formulas (7), (8) are valid. But many equalities turn into inclusions:

$$
\mathscr{H}_{p} \subset \operatorname{ran} Q^{*} ; \quad J \mathscr{H}_{1} \subset \operatorname{ker} Q^{*} ; \quad \mathscr{H}_{1} \subset \mathscr{H}_{d}^{[\perp]}=\mathscr{H}_{p}^{\perp} .
$$

Also the sets $\left(\mathscr{H}_{p} \oplus \mathscr{H}_{d}\right)^{\perp}, \operatorname{ker} Q \cap \operatorname{ker} Q^{*}, \mathscr{H}_{1} \cap J \mathscr{H}_{1}$ are now all different from each other, in the general case, and so different definitions of $\mathscr{H}_{s}$ are possible. Desiring to preserve the properties $\mathscr{H}_{s} \subset \mathscr{H}_{1}$ and $\mathscr{H}_{s}=J \mathscr{H}_{s}$ we are left with the choice $\mathscr{H}_{s}=\mathscr{H}_{1} \cap J \mathscr{H}_{1}$. In this case one has instead of (10) and (11):

$$
\overline{\mathscr{H}}_{p} \oplus \overline{\mathscr{H}}_{d} \oplus \mathscr{H}_{s} \subset \mathscr{H} ; \quad \overline{\mathscr{H}}_{d} \oplus \mathscr{H}_{s}=\overline{\mathscr{H}}_{d}[+] \mathscr{H}_{s} \subset \mathscr{H}_{1} .
$$

In general, only the implications $1 \leftarrow 2 \rightarrow 3 \rightarrow 4$ survive in Proposition 2 .

Apart from $Q$, the structure of the state space of a BRST theory is determined by the ghost number operator $Q_{c}$. In the general theory it is natural to define it in a way similar to $Q$, by means of a certain derivation $\delta_{c}$ on the $\operatorname{Op} J^{*}$-algebra $(\mathscr{P}, \mathscr{D})$ : for any monomial $A \in \mathscr{P}$ we put

$$
\delta_{c}(A)=n(A) A,
$$

and we define $Q_{c}$ as the generator of this derivation, according to (2) and (3):

$$
Q_{c} A \Omega=-i \delta_{c}(A) \Omega \text {. }
$$

As was the case with $Q$, the existence of $Q_{c}$ is a restriction on the theory. Let us denote $\mathscr{P}_{n}$ the linear hull of all monomials $A \in \mathscr{P}$ with $n(A)=n$ and split $\mathscr{P}$ and $\mathscr{D}$ into sectors with a given $n$ :

$$
\mathscr{P}=L\left\{\mathscr{P}_{n} \mid n \in \mathbb{Z}\right\} ; \quad \mathscr{D}=L\left\{\mathscr{L}_{n} \mid n \in \mathbb{Z}\right\} ; \quad \mathscr{L}_{n} \equiv \mathscr{P}_{n} \Omega .
$$


Then (12) is a correct definition of $Q_{c}$ if and only if

$$
\mathscr{L}_{n} \cap \mathscr{L}_{m}=\{0\}, \quad m, n \in \mathbb{Z}, \quad m \neq n .
$$

Examples when (13) is broken are presented in [10]. If $Q_{c}$ does exist, then there also exist [10] the ghost conjugation operator $U_{c}$ which is a $J$-hermitian and $J$-unitary operator such that

$$
\left\{U_{c}, Q_{c}\right\}=0, \quad U_{c} \mathscr{L}_{n}=\mathscr{L}_{-n}, \quad U_{c} \Omega=\Omega .
$$

Due to these properties, it is often convenient to use $U_{c}$ as the metric operator $J$. In addition, the following conditions are equivalent $[10]$ :

1. (12) defines the $J$-symmetric operator $Q_{c}$ on $\mathscr{D}$;

2. $\left\langle\Omega, \delta_{c}(A) \Omega\right\rangle=0$ for all $A \in \mathscr{P}$;

3. $\mathscr{L}_{n} \subset \mathfrak{M}_{0}, n \neq 0, \mathscr{L}_{n}[\perp] \mathscr{L}_{m}, m \neq-n$.

If these conditions are met, $Q_{c}$ has a purely discrete and imaginary spectrum, $\sigma\left(Q_{c}\right)=i \mathbb{Z}$, and the root lineals $\mathscr{L}_{n}$ (principal subspaces in the terminology of [9]); the lineals $\mathscr{L}_{0}$ and $\mathscr{L}_{n} \dot{+} \mathscr{L}_{-n}$ are nondegenerate and $\mathscr{H}$ has the decomposition

$$
\mathscr{H}=\overline{\mathscr{L}_{0}\left[+\underset{n=1}{\infty}\left(\mathscr{L}_{n} \dot{+} \mathscr{L}_{-n}\right)\right.} \text {. }
$$

When $\mathscr{H}_{1}$ is non-negative, it is considered as the prephysical subspace of BRST theory, and one takes as the space of true physical states the (Hilbert) space of BRST cohomologies $\mathscr{H}_{1} / \mathscr{H}_{d}$ which is isomorphic to $\mathscr{H}_{s}$ due to (11). According to Proposition 2, $\mathscr{H}_{s}$ does not include any states with the fixed non-zero ghost number, i.e.

$$
\mathscr{H}_{s} \cap \mathscr{L}_{n}=\{0\} \text { for any } n \neq 0 .
$$

Taking into account $1 \rightarrow 2$ in Proposition 2 we obtain

Corollary 3. In the BRST theory with the non-negative kernel of the BRST charge all the BRST cohomologies with the non-zero ghost numbers are trivial, i.e. for any $\Phi \in \mathscr{H}_{1} \cap \mathscr{L}_{n}, n \neq 0$ one has $[\Phi]=0$, where $[\Phi]$ is the equivalence class of $\Phi$ in $\mathscr{H}_{1} / \mathscr{H}_{d}$.

The algebraic structure of the theory is fixed completely when the commutation relation between $Q$ and $Q_{c}$ is given. In concrete theories this relation has to be derived; in a general theory we can only give heuristic arguments. As a charge, $Q$ must increase the number of ghosts $n_{c}(\Phi)$ in a state $\Phi$, i.e. $n_{c}(Q \Phi)=n_{c}(\Phi)+1$, whence

$$
\left[i Q_{c}, Q\right]=Q \text {. }
$$

Hence it is clear that in a theory symmetrical in ghosts and antighosts, there should be another charge $\bar{Q}$ similar to $Q$ but increasing the number of antighosts so that $n_{c}(\bar{Q} \Phi)=n_{c}(\Phi)-1$, i.e.

$$
\left[i Q_{c}, \bar{Q}\right]=-\bar{Q} \text {. }
$$

The pair of $J$-symmetric operators $Q, Q_{c}$ satisfying the conditions $(15)$ and $Q^{2}=0$ is called the BRST algebra; the triple $\left(Q, \bar{Q}, Q_{c}\right)$ satisfying the conditions (15), (16), $Q^{2}=0, \bar{Q}^{2}=0$ and $\{Q, \bar{Q}\}=0$ (there is no simple intuitive interpretation for the last condition) is called the extended BRST algebra. Structure of $\mathscr{H}$ as the space of a 
representation of the BRST algebra is the superposition of the " $Q$-decompositions" (10) and (11) and the " $Q_{c}$-decomposition" (14) connected by (15).

From (15) one gets readily

$$
Q \mathscr{L}_{n} \subset \mathscr{L}_{n+1}, \quad Q_{c} \mathscr{H}_{1} \subset \mathscr{H}_{1}, \quad Q_{c} \mathscr{H}_{d} \subset \mathscr{H}_{d} .
$$

But the singlet subspace $\mathscr{H}_{s}$ is, in general, invariant for $Q_{c}$ only under some additional condition. One of conditions which will do is the choice of a metric operator with the property $\left\{J, Q_{c}\right\}=0$, in particular, the "physical" choice $J=U_{c}$. With this choice we have

$$
Q_{c} \mathscr{H}_{s} \subset \mathscr{H}_{s}
$$

as well as equivalent relationships

$$
Q_{c} \mathscr{H}_{p} \subset \mathscr{H}_{p}, \quad Q_{c}^{*} \mathscr{H}_{1} \subset \mathscr{H}_{1}, \quad Q_{c}^{*} \mathscr{H}_{d} \subset \mathscr{H}_{d} .
$$

(If $\operatorname{dom} Q \supset \mathscr{D}$, then we must put in these formulas instead of $\mathscr{H}_{1}, \mathscr{H}_{p}, \mathscr{H}_{d}, \mathscr{H}_{s}$ their intersections with $\mathscr{D}$.) It is easy to describe in a similar way the representation space of the extended BRST algebra.

It happens sometimes in physical models that $\mathscr{H}_{1}$ is not non-negative while a narrower subspace $\mathscr{H}_{1} \cap \mathscr{L}_{0}$ or $\mathscr{H}_{1} \cap \operatorname{ker} \bar{Q}$ enjoys this property (and is accepted as a prephysical subspace instead of $\mathscr{H}_{1}$ ). It would be of interest to find some general conditions for this. For example, it is easy to show that $\mathscr{H}_{1} \cap \operatorname{ker} \bar{Q}$ is non-negative if $\mathscr{H}_{d}+\mathscr{H}_{\bar{d}}, \mathscr{H}_{\bar{d}} \equiv \operatorname{ran} \bar{Q}$ is a maximal neutral lineal.

Acknowledgements. We would like to thank T. Ya. Azizov and V. P. Pavlov for useful discussions.

\section{References}

1. Nishijima, K.: Representations of BRS algebra. Nucl. Phys. B 238, 601-620 (1984)

2. Kugo, T., Ojima, I.: Local covariant operator formalism in non-abelian gauge theories and quark confinement problem. Suppl. Prog. Theor. Phys. 66 (1979)

3. Dadashyan, K.Yu., Horuzhy, S.S.: On field algebras in quantum field theory with indefinite metrics. Theor. Math. Phys. 54, 35-47 (1983)

4. Nakanishi, N.: A new way of describing the Lie algebras encountered in quantum field theory. Prog. Theor. Phys. 60, 284-294 (1978)

5. Strocchi, F.: Local and covariant gauge quantum field theory. Cluster property, superselection rules and the infrared problem. Phys. Rev. D 17, 2010-2022 (1978)

6. Ojima, I., Hata, H.: Observables and quark confinement in the covariant canonical formalism of Yang-Mills theory. II. Z. Phys. C 1, 405-412 (1979)

7. Danford, N., Schwartz, J.T.: Linear operators. Vol. I. New York, London, Sydney, Toronto: Wiley 1971

8. Azizov, T.Ya., Iokhvidov, I.S.: Foundations of the theory of linear operators in indefinite metric spaces. Moscow: Nauka 1986 (in Russian)

9. Bognar, J.: Indefinite inner product spaces. Berlin, Heidelberg, New York: Springer 1974

10. Azizov, T.Ya., Horuzhy, S.S.: On operators of ghost number and ghost conjugation in the BRST quantization framework. Theor. Math. Phys. 80, No. 1 (1989) (in press)

Communicated by L. Alvarez-Gaumé

Received August 1, 1988; in revised form January 13, 1989 
